\section{Clinical efficacy of ruxolitinib and chemotherapy in a child with Philadelphia chromosome-like acute lym- phoblastic leukemia with GOLGA5-JAK2 fusion and induction failure}

Patients with Philadelphia chromosome-like B-cell lymphoblastic leukemia (Ph-like or BCR-ABL1-like BALL) experience high relapse rates and are difficult to cure with conventional chemotherapy., ${ }^{1,2}$ The Ph-like ALL subtype comprises $15-25 \%$ of B-ALL in older children and adolescents/young adults (AYAs) and 20-40\% of cases in older adults. ${ }^{1,2} \mathrm{Ph}$-like ALL is driven by alterations in cytokine receptor signaling pathway genes that induce oncogenic kinase signaling, suggesting therapeutic potential for kinase inhibitors. ${ }^{1}$ Approximately $50 \%$ of $\mathrm{Ph}$-like ALL has rearrangements in CRLF2 (cytokine receptor-like factor 2), often with concomitant JAK2 or JAK1 point mutations, which leads to constitutive JAK/STAT signaling targetable by JAK inhibitors (JAKi) such as ruxolitinib. JAK2 and EPOR translocations comprise an additional $8-12 \%$ of $\mathrm{Ph}$-like ALL in children/AYAs and adults.1-3 JAK2- or EPOR-rearranged ALLs are also characterized by hyperactive JAK/STAT signaling and are potentially very sensitive to JAK inhibition. ${ }^{3,4}$ While JAKi addition to multi-agent chemotherapy is under current clinical investigation in children and adults with newlydiagnosed or relapsed CRLF2-R/JAK-mutant Ph-like ALL via early-phase trials (clinicaltrials.gov identifier 02723994, 03117751, 02420717), whether this therapeutic strategy
A

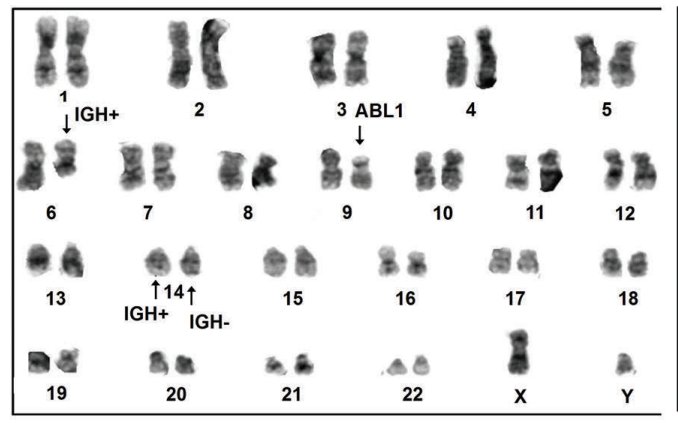

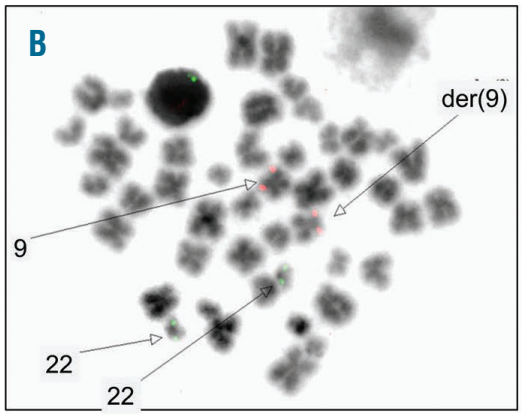
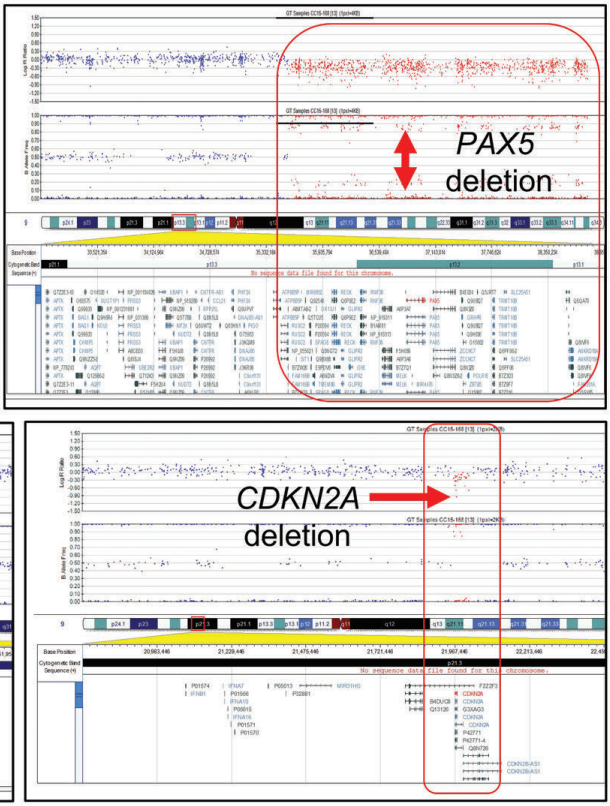

D

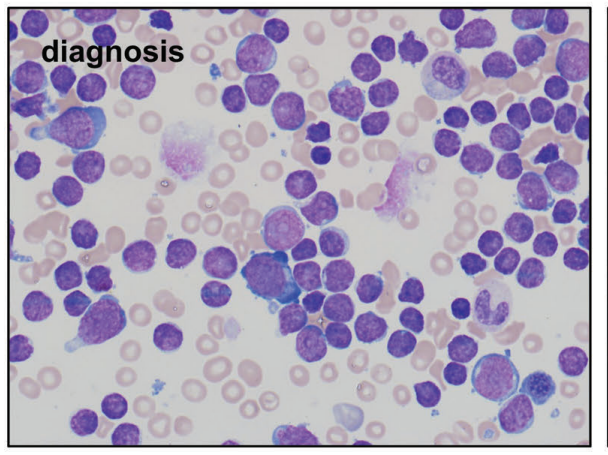

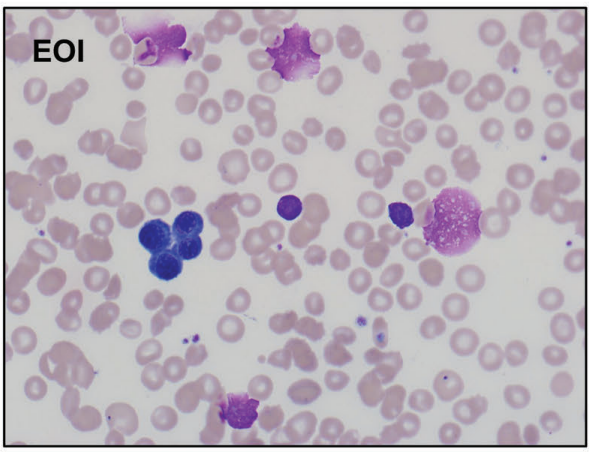

Figure 1. Clinical cytogenetic and fluorescence in situ hybridization (FISH) analyses of the patient's diagnostic bone marrow aspirate. (A) Karyotype shows multiple cytogenetic abnormalities, including abnormalities of chromosome 9 and $t(6 ; 14)$ involving $I G H$ and an unknown fusion partner on chromosome 6. (B) FISH analysis demonstrates absence of $B C R-A B L 1$ translocation with two normal signals for $A B L 1$ on chromosome 9 (red probes) and BCR on chromosome 22 (green probes). (C) Single nucleotide polymorphism (SNP) array analysis of the patient's diagnostic bone marrow aspirate demonstrates partial duplication of JAK2 and deletions of IKZF1, PAX5, and CDKN2A. (D) Photomicrographs of diagnostic (left) and end-of-induction (EOI, right) Wright-Giemsastained bone marrow aspirates. Images were captured at $100 X$ via an Olympus BX53 microscope and UPlanSApo camera using CellSens Standard software. End of induction marrow blasts are markedly smaller and have a normal hematogone-like appearance by morphology. 


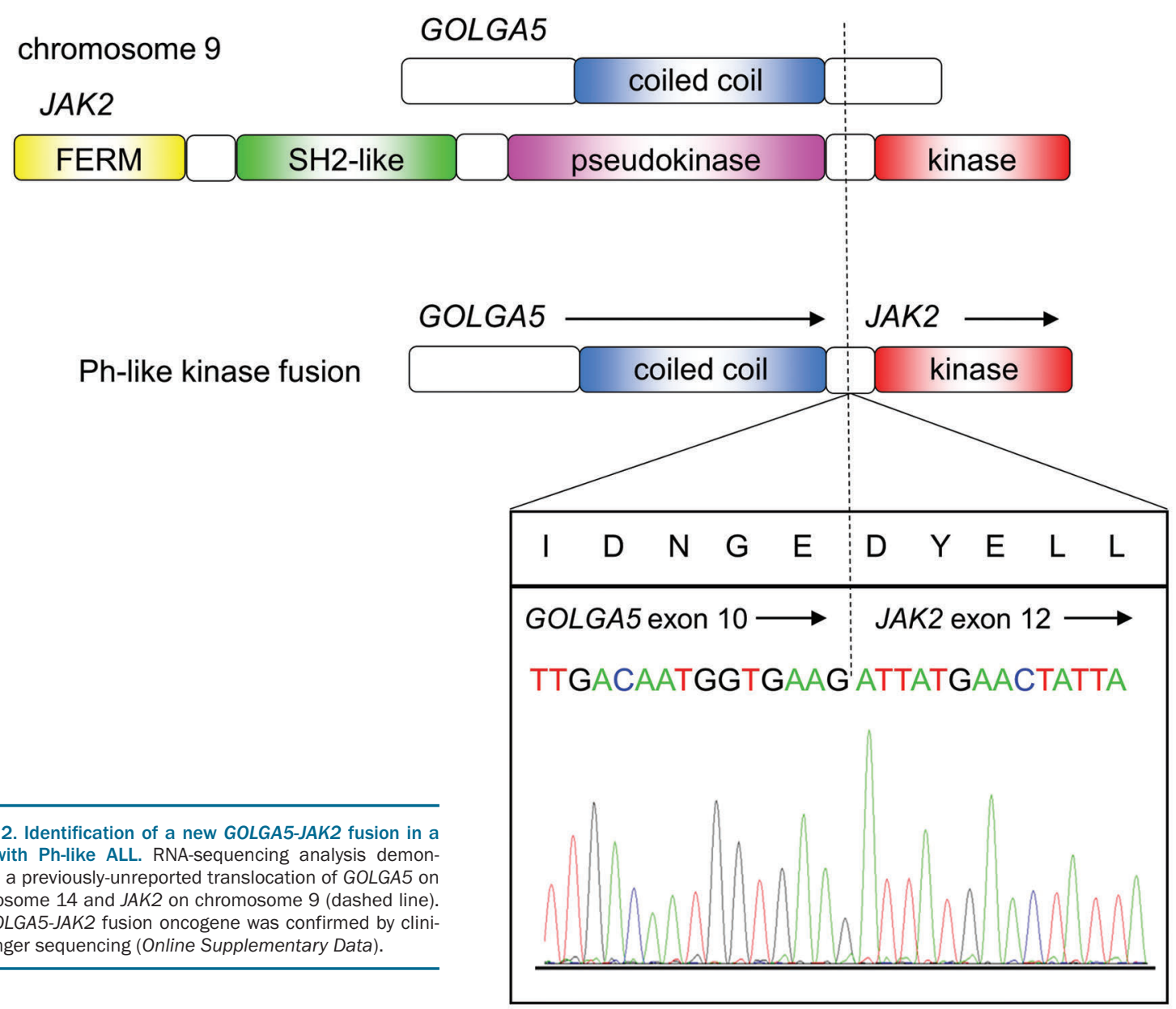

will decrease relapse and improve survival is not yet known. We report an exceptional case of a child with chemoresistant $\mathrm{Ph}$-like ALL and induction failure in whom we identified a novel GOLGA5-JAK2 fusion. We further demonstrate molecular clearance of the GOLGA5-JAK2 fusion and leukemia remission induction after addition of ruxolitinib to high-risk B-ALL chemotherapy.

A 10-year-old Caucasian male was diagnosed with National Cancer Institute high-risk B-ALL in June 2015 after presenting with fatigue, weight loss, and dyspnea. Physical examination was pertinent for pallor, orthopnea, and hepatosplenomegaly. Diagnostic laboratory studies showed white blood cell (WBC) count $578,000 / \mu \mathrm{L}$ with $92 \%$ blasts, hemoglobin $5.7 \mathrm{~g} / \mathrm{dL}$, and platelets $89,000 / \mu \mathrm{L}$. His dyspnea and hypoxia improved following emergent leukopheresis.

Bone marrow aspirate analysis demonstrated B-ALL (CD45+/CD9 ${ }^{+} / \mathrm{CD}_{10} / \mathrm{CD}^{+} 19^{+} / \mathrm{CD} 20$ (subset) ${ }^{+} / \mathrm{CD} 34^{+} / \mathrm{cCD}$

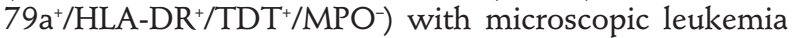
involvement of the cerebrospinal fluid (CNS2a). The patient's parents provided informed consent for research specimen banking on the Children's Oncology Group (COG) AALL08B1 study and induction chemotherapy on the COG AALL1131 trial (clinicaltrials.gov identifier 02883049; Online Supplementary Methods). Cytogenetic analysis demonstrated a complex karyotype with $46, \mathrm{X} \mathrm{Y}, \mathrm{d}$ e r $(6) \mathrm{t}(6 ; 14)(\mathrm{p} 23 ; \mathrm{q} 32) \mathrm{d}$ e 1 ( 6 ) ( q 15 q 23 ), d e r (9) inv (9) ( p ? q ? 34 ) d e l (9) (p21p13)del(9)(q22q31),der(14)t(6;14)(p23;q32)[14].ish,d
er(6)(IGH+), der(14)(IGH-)/46,XY[4]. Fluorescence in situ hybridization studies were negative for $B C R-A B L 1$, ETV6-RUNX1, and IGH-MYC translocations and CRLF2, KMT2A (MLL), NUP98, and PDGFRB rearrangements (Figure 1A-B). Single nucleotide polymorphism array analysis demonstrated chromosome 9 duplication involving JAK2 and deletions of IKZF1, PAX5, and CDKN2A concordant with the recently-described $I K Z F 1^{\text {(plus) }}$ subtype (Figure 1C, Online Supplementary Table S1). ${ }^{5}$ The child experienced poor initial response to chemotherapy with $43.3 \%$ peripheral blood minimal residual disease (MRD) on induction day 8. While his induction day 29 bone marrow appeared morphologically normal (M1 remission), flow cytometry (FC) analysis revealed $40 \%$ $M R D$, consistent with induction failure (Figure 1D).

The patient's high WBC count, IKZF1 deletion, absence of $B C R-A B L 1$ fusion, and induction failure raised significant suspicion for Ph-like ALL. Low-density microarray (LDA) analysis of leukemia RNA demonstrated a very high Ph-like ALL expression signature (0.835 8gene and 0.987 15-gene scores) and normal CRLF2 expression. ${ }^{6}$ However, clinical testing for 39 known $\mathrm{Ph}$ like kinase fusions via reverse-transcriptase polymerase chain reaction (RT-PCR) was negative for $C R L F 2$, $J A K 2, A B L 1, A B L 2, C S F 1 R$, and PDGFRB fusions and $J A K 1$ and $J A K 2$ point mutations. ${ }^{6}$ Given the patient's lack of CRLF2 rearrangement and LDA score $>0.8$ (associated with ABL-class kinase fusions in $>80 \%$ of cases $^{6}$ ) and reported $E B F 1-P D G F R B$ fusions in patients with $\mathrm{Ph}$-like ALL induction failure, ${ }^{7}$ dasatinib $60 \mathrm{mg} / \mathrm{m}^{2}$ orally once- 
A

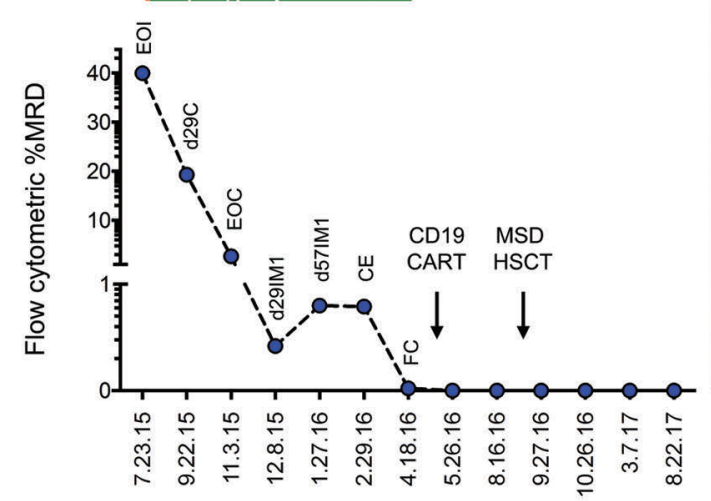

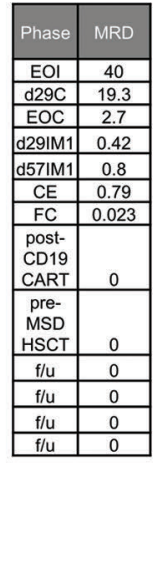

B

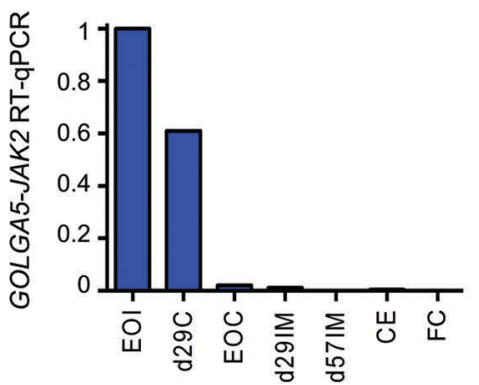

C

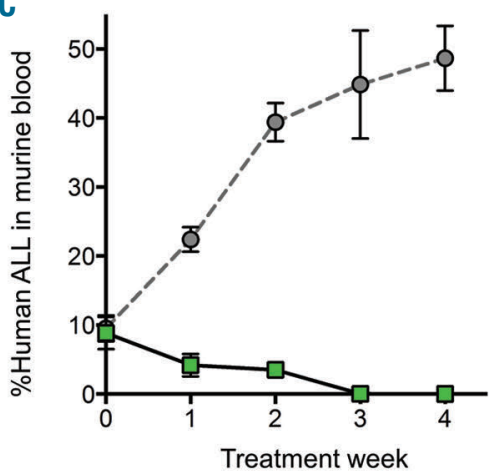

D

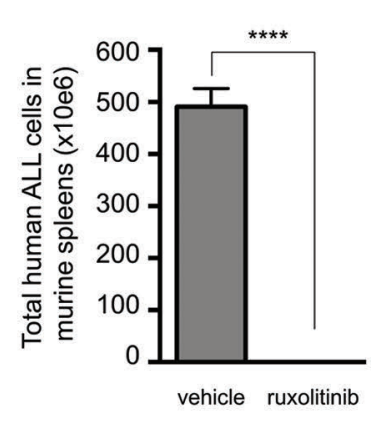

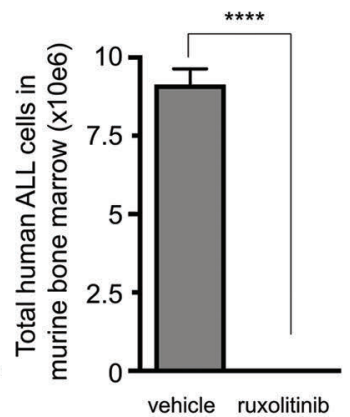

Figure 3. Clinical response to JAK inhibition and chemotherapy and assessment of in vivo efficacy of ruxolitinib monotherapy in GOLGA5-JAK2 Ph-like ALL patient-derived xenograft (PDX) model. (A) Flow cytometric minimal residual disease (MRD) monitoring of patient's bone marrow during combined ruxolitinib and AALL1131-type chemotherapy demonstrates rapid induction of leukemia remission (y-axis and table inset; assay sensitivity $\left.1 \times 10^{4}\right)$. X-axis indicates dates of bone marrow aspirate assessments. Orange bar = dasatinib $60 \mathrm{mg} / \mathrm{m}^{2}$ orally once daily. Green bar $=$ ruxolitinib $40 \mathrm{mg} / \mathrm{mg} /$ dose orally twice daily. EOI: end of induction; d29C: day 29 of consolidation; EOC: end of consolidation; IM1: interim maintenance 1; CE: cyclophosphamide/etoposide; FC : fludarabine/cyclophosphamide; CD19 CART: CD19-redirected chimeric antigen receptor T-cell immunotherapy; MSD HSCT: matched sibling donor allogeneic hematopoietic stem cell transplantation; f/u: follow-up time points. (B) Quantitative reverse-transcriptase and real-time polymerase chain reaction (RT-qPCR) monitoring of the GOLGA5-JAK2 fusion transcript in serial bone marrow aspirate specimens demonstrated rapid molecular clearance of JAK2-mutant ALL with combination therapy as in (A) (assay sensitivity $1 \times 10^{4}-1 \times 10^{5}$ ). (C) Eight-week-old female NSG mice were injected via tail vein with $2 \times 10^{6}$ of the child's GOLGA5-JAK2 ALL cells from diagnostic bone marrow (PAXDBJ) to create primary xenograft models, and murine spleens were harvested for flow cytometry (FC) quantification of $\mathrm{CD} 10^{+} \mathrm{CD} 19^{+} \mathrm{CD}_{4} 5^{+}$human B-ALL cells and subsequent xenotransplantation. Secondary PDX models were created via injection of 1 x $10^{6}$ viably cryopreserved murine splenocytes from well-engrafted primary PDX mice. After $5 \%$ human B-ALL engraftment was detected in murine peripheral blood by FC, secondary PDX mice were randomized to treatment with control or ruxolitinib $2 \mathrm{~g} / \mathrm{kg}$ chow continuously provided for 28 days ( $\mathrm{n}=10$ mice/cohort). Rapid clearance of human leukemia cells was observed in murine peripheral blood with ruxolitinib treatment by weekly quantitative FC analysis. (D) Ruxolitinib potently inhibited leukemia proliferation in vivo with near-complete elimination of human ALL in murine spleens and bone marrow after 4 weeks of treatment $(* * * * P<0.0001$ by Student's t-test).

daily was empirically added to the child's AALL1131type consolidation therapy (Online Supplementary Methods). We then performed research-level RNAsequencing and identified a previously-unreported GOLGA5-JAK2 fusion from $\mathrm{t}(9 ; 14)$ (Figure 2), which was confirmed by clinical Sanger sequencing and Archer anchored multiplex PCR FusionPlex testing.

Given our prior demonstrated sensitivity of Ph-like ALL patient-derived xenograft (PDX) models with JAK2 fusions to ruxolitinib, ${ }^{4}$ dasatinib was discontinued at consolidation day 7 upon detection of the GOLGA5-JAK2 fusion. Ruxolitinib was started at $40 \mathrm{mg} / \mathrm{m}^{2} /$ dose orally twice-daily (PO BID), a safe and pharmacodynamicallyactive dose identified on the COG ADVL1011 trial ${ }^{8}$ and the starting dose on the now-open COG AALL1521/INCB18424-269 trial (clinicaltrials.gov identifier 02723994). The patient tolerated combined ruxolitinib and AALL1131-type post-induction chemotherapy well. Ruxolitinib was briefly discontinued after consolidation for grade 4 (CTCAE v4.0) transaminitis of unclear etiology that resolved in $<7$ days and empirically between myelosuppressive chemotherapy phases to facilitate blood count recovery. He did not experience prolonged cytopenias or toxicities beyond those typically observed with AALL1131 backbone therapy. Serial bone marrow aspiration with FC MRD testing was performed to assess response. GOLGA5-JAK2 fusion transcript levels were followed in marrow specimens by quantitative RT-PCR assays (Online Supplementary Methods).

Sequential marrow analysis demonstrated significant clinical response to combined ruxolitinib/chemotherapy by the end of consolidation and interim maintenance 1 (IM1), although with persistent $\mathrm{MRD} \geq 0.01 \%$ by FC (Figure 3A). Given high relapse risk and poor outcomes of children with MRD positivity prior to allogeneic hematopoietic stem cell transplantation (HCST), 9,10 the child received autologous CD19-redirected chimeric antigen receptor T cells (CD19CART) in April 2016 on a 
phase 1 trial (clinicaltrials.gov identifier 02374333) and achieved MRD-negative remission. Given his prior chemoresistance and known relapse risk postCD19CART, he underwent matched sibling donor HSCT in August 2016 following myeloablative conditioning. He remains in continued $\mathrm{MRD}$-negative leukemia remission with full donor chimerism at 20 months post-HSCT.

Research-level RT-PCR monitoring of GOLGA5-JAK2 transcript levels in the patient's bone marrow demonstrated near-complete clearance of the Ph-like ALL fusion by the end of consolidation and undetectable transcript levels at the end of IM1 (Figure 3B), although he had persistent low-level MRD detected by clinical FC analyses. MRD quantification by currently-available next-generation sequencing or PCR-based immunoglobulin/T-cell receptor rearrangement methodologies may be beneficial in such situations to increase precision of MRD assessments and to inform therapeutic decisions.

Simultaneously, we established a PDX model (PAXDBJ) using viably cryopreserved diagnostic bone marrow ALL cells from the child engrafted into NOD.CgPrkdcscid Il2rgtm1Wj1/SzJ (NSG) mice via informed consent on Institutional Review Board- and Institutional Animal Care and Use Committee-approved research protocols as described. ${ }^{4,11}$ Once $\geq 1 \%$ human ALL was detected in murine peripheral blood, secondary PDX mice were randomized to control or ruxolitinib $2 \mathrm{~g} / \mathrm{kg}$ chow (a gift from the Incyte Corporation) treatment continuously provided. Human ALL cells were quantified weekly by FC analysis of retro-orbital murine venous blood samples. Total human ALL burden was enumerated in spleens of sacrificed animals after 28 days of treatment. ${ }^{11}$ Data were compared by t-tests using Prism (GraphPad). GOLGA5-JAK2 ALL PDX models treated with ruxolitinib demonstrated rapid clearance of human leukemia in murine peripheral blood. Residual ALL was effectively undetectable by quantitative FC analysis of murine spleens and bone marrow after 28 days of ruxolitinib versus control chow $(\mathrm{P}<0.0001)$ (Figure $3 \mathrm{C}-\mathrm{D})$. These data demonstrate a similar magnitude and rate of ruxolitinibinduced leukemia clearance as was observed in the patient, suggesting that preclinical PDX models may predict clinical responses to JAKi.

Prior studies have demonstrated remarkable outcome improvements in children with $B C R-A B L 1$-rearranged $\left(\mathrm{Ph}^{+}\right)$ALL with imatinib addition to chemotherapy. ${ }^{12,13}$ Integration of kinase inhibitor-based approaches for patients with Ph-like ALL may similarly decrease relapse risk and improve survival given analogous hyperactivation of kinase signaling. While recently-opened clinical trials are investigating efficacy of ruxolitinib and dasatinib in patients with JAK pathway-mutant and $A B L$ class-mutant $\mathrm{Ph}$-like ALL, respectively, anecdotal use of kinase inhibitors in Ph-like ALL has primarily been reported in children/AYA with ABL class alterations treated with imatinib or dasatinib. ${ }^{1}$ To our knowledge, only one report has described ruxolitinib use in a patient with Ph-like ALL. ${ }^{14}$ In that study, a 17-year-old Native American female with CRLF2-overexpressing Ph-like ALL and a JAK2 F694L point mutation had increasing MRD $(10.4 \%)$ after consolidation chemotherapy. Ruxolitinib $40 \mathrm{mg} / \mathrm{m} 2 /$ dose PO BID was added to AALL1131-type interim maintenance and delayed intensification therapy. She achieved MRD $<0.01 \%$ with JAKi/chemotherapy, underwent allogeneic HSCT, and was in continued remission at 100 days post-HSCT. ${ }^{14}$

Our concordant clinical data demonstrate safe and clinically effective combination of high-dose ruxolitinib and post-induction multi-agent chemotherapy in a child with
Ph-like ALL with a JAK2 fusion and induction failure, which has been associated with dismal long-term outcomes. ${ }^{7,15}$ We observed excellent reduction in the patient's ALL burden and significantly decreased GOLGA5-JAK2 fusion levels with JAKi/chemotherapy, although persistence of FC-detectable MRD prompted us to pursue subsequent cellular immunotherapy and HSCT. More importantly, our patient's case highlights continued discovery of new Ph-like alterations and need for unbiased genetic testing approaches to diagnose fusions in real-time for potential clinical intervention. Anchored multiplex PCR fusion assays and RNA-sequencing technologies may provide an appreciable detection advantage over current PCR-based clinical assays for known fusions. ${ }^{6}$

In summary, (1) early clinical suspicion for Ph-like ALL and (2) rapid RNA-sequencing analysis capabilities facilitated identification of a previously-unknown and initially clinically-unidentifiable GOLGA5-JAK2 fusion in a child with significant leukocytosis and induction failure. This timely genetic characterization allowed swift implementation of commercially-available JAKi therapy (prior to current clinical trials), which was well-tolerated with an intensive chemotherapy backbone and induced morphologic remission. Concomitant PDX modeling demonstrating exquisite in vivo sensitivity of GOLGA5-JAK2 ALL cells to ruxolitinib provided additional biologic validation of the child's clinical response. Data from AALL1521 and other trials will determine the potential efficacy of combining ruxolitinib and chemotherapy in larger cohorts of patients with JAK pathway-mutant Phlike ALL.

Yang Y. Ding, ${ }^{1,2}$ Julie W. Stern, ${ }^{1,2}$ Tracey F. Jubelirer, ${ }^{1,2}$ Gerald B. Wertheim, ${ }^{3}$ Fumin Lin, ${ }^{4}$ Fengqi Chang, ${ }^{4}$ Zhaohui Gu, Charles G. Mullighan, ${ }^{5}$ Yong Li, Richard C. Harvey, ${ }^{6}$ I-Ming Chen, ${ }^{6}$ Cheryl L. Willman, Stephen P. Hunger, Marilyn M. Lit and Sarah K. Tasian ${ }^{1,2}$

${ }^{1}$ Children's Hospital of Philadelphia, Division of Oncology and Center for Childhood Cancer Research, PA; ${ }^{2}$ University of Pennsylvania Perelman School of Medicine, Department of Pediatrics, $P A ;{ }^{3}$ University of Pennsylvania Perelman School of Medicine, Department of Pathology and Laboratory, PA, ${ }^{4}$ Children's Hospital of Philadelphia, Division of Genomic Diagnostics Medicine; Children's Hospital of Philadelphia, Division of Hematopathology PA; ${ }^{5} S t$ Jude Children's Research Hospital, Department of Pathology, Memphis, $T N_{i}{ }^{6}$ University of New Mexico, Department of Pathology and Hematologic Malignancies Research Program, Albuquerque, NM, USA.

Acknowledgments: we thank Dr. Bruce Ruggeri at the Incyte Corporation for scientific collaboration and generous provision of ruxolitinib chow for preclinical studies.

Funding: these studies were supported by the National Institutes of Health/National Child Health and Human Development award T32HD043021 (YYD), National Cancer Institute K08CA184418 award (SKT), and the Rally Foundation for Childhood Cancer Research (SKT). CGM is the William E Evans Endowed Chair in Pathology. CLW is the Maurice and Marguerite Liberman Distinguished Chair in Cancer Research Professor of Pathology and Medicine. SPH is the Jeffrey E Perelman Distinguished Chair in Pediatrics at The Children's Hospital of Philadelphia.

Correspondence: tasians@email.chop.edu doi:10.3324/haematol.2018.192088

Information on authorship, contributions, and financial \& other disclosures was provided by the authors and is available with the online version of this article at www. haematologica.org. 


\section{References}

1. Roberts KG, Li Y, Payne-Turner D, et al. Targetable kinase-activating lesions in Ph-like acute lymphoblastic leukemia. N Engl J Med. 2014;371(11):1005-1015.

2. Roberts KG, Gu Z, Payne-Turner D, et al. High frequency and poor outcome of philadelphia chromosome-like acute lymphoblastic leukemia in adults. J Clin Oncol. 2017;35(4):394-401.

3. Iacobucci I, Li Y, Roberts KG, et al. Truncating erythropoietin receptor rearrangements in acute lymphoblastic leukemia. Cancer Cell. 2016;29(2):186-200.

4. Maude SL, Tasian SK, Vincent T, et al. Targeting JAK1/2 and mTOR in murine xenograft models of $\mathrm{Ph}$-like acute lymphoblastic leukemia. Blood. 2012;120(17):3510-3518.

5. Stanulla M, Dagdan E, Zaliova M, et al. IKZF1(plus) defines a new minimal residual disease-dependent very-poor prognostic profile in pediatric B-cell precursor acute lymphoblastic leukemia. J Clin Oncol. 2018;36(12):1240-1249.

6. Reshmi SC, Harvey RC, Roberts KG, et al. Targetable kinase gene fusions in high risk B-ALL: a study from the Children's Oncology Group. Blood. 2017;129(25):3352-3361.

7. Schwab C, Ryan SL, Chilton L, et al. EBF1-PDGFRB fusion in pediatric B-cell precursor acute lymphoblastic leukemia (BCP-ALL): genetic profile and clinical implications. Blood. 2016;127(18):22142218.

8. Loh ML, Tasian SK, Rabin KR, et al. A phase 1 dosing study of ruxolitinib in children with relapsed or refractory solid tumors, leukemias, or myeloproliferative neoplasms: A Children's Oncology
Group phase 1 consortium study (ADVL1011). Pediatr Blood Cancer. 2015;62(10):1717-1724.

9. Bader P, Kreyenberg H, Henze GH, et al. Prognostic value of minimal residual disease quantification before allogeneic stem-cell transplantation in relapsed childhood acute lymphoblastic leukemia: the ALLREZ BFM Study Group. J Clin Oncol. 2009;27(3):377-384.

10. Pulsipher MA, Carlson C, Langholz B, et al. IgH-V(D)J NGS-MRD measurement pre- and early post-allotransplant defines very lowand very high-risk ALL patients. Blood. 2015;125(22):3501-3508.

11. Tasian SK, Teachey DT, Li Y, et al. Potent efficacy of combined $\mathrm{PI} \mathrm{K} / \mathrm{mTOR}$ and JAK or ABL inhibition in murine xenograft models of Ph-like acute lymphoblastic leukemia. Blood. 2017;129(2):177187.

12. Biondi A, Schrappe M, De Lorenzo P, et al. Imatinib after induction for treatment of children and adolescents with Philadelphia-chromosome-positive acute lymphoblastic leukaemia (EsPhALL): a randomised, open-label, intergroup study. Lancet Oncol. 2012;13(9):936-945.

13. Schultz KR, Carroll A, Heerema NA, et al. Long-term follow-up of imatinib in pediatric Philadelphia chromosome-positive acute lymphoblastic leukemia: Children's Oncology Group study AALL0031. Leukemia. 2014;28(7):1467-1471.

14. Mayfield JR, Czuchlewski DR, Gale JM, et al. Integration of ruxolitinib into dose-intensified therapy targeted against a novel JAK2 F694L mutation in B-precursor acute lymphoblastic leukemia. Pediatr Blood Cancer. 2017;64(5).

15. Schrappe M, Hunger SP, Pui CH, et al. Outcomes after induction failure in childhood acute lymphoblastic leukemia. N Engl J Med. 2012;366(15):1371-1381. 